\title{
Influence of Autonomous Vehicles on Freeway Traffic Performance for Undersaturated Traffic Conditions
}

\author{
By Osama ElSahly ${ }^{*} \&$ Akmal Abdelfatah ${ }^{ \pm}$
}

\begin{abstract}
Autonomous vehicles (AVs) are smart driving technology that is expected to alter the perception of transportation. The purpose of this study is to evaluate the impacts of AVs on freeway traffic performance at different percentages of $A V s$ ranging from $0 \%$ to $100 \%$ and at two different undersaturated traffic volume levels with demand to capacity ratios of 0.6 and 0.8. The well-known VISSIM software was used to develop a microsimulation model to evaluate different scenarios that represent different market penetration rates of AVs and different demand to capacity ratios. The results showed that the minimum improvement was at 5\% AVs and 0.6 demand to capacity ratio and the maximum improvement was achieved at $100 \%$ AVs and 0.8 demand to capacity ratio. The increase in the average speed ranges from about $5 \%$ to about $15 \%$, the reduction in travel time ranges from about $1 \%$ to about $12 \%$ and the delay reduction is about $18 \%$ to about $97 \%$. The improvement in traffic performance when AVs market penetration rates increases from $0 \%$ to $100 \%$ is attributed to the fact that the conventional vehicles $(C V s)$ are replaced by $A V s$ that can travel with higher constant speed and with a smaller time headway. Statistical t-test was carried out to examine the statistical significance of the difference between scenarios' average speeds and between the average speed of both AVs and CVs. The test revealed that there average speed values of AVs are significantly higher than $C V s$ values for all AVs market penetration rates at demand to capacity ratios of 0.6 and 0.8. Because at these demand to capacity ratios the congestion is low. Thus, AVs can travel freely with speeds significantly higher than CVs.
\end{abstract}

Keywords: Autonomous vehicles, Freeway performance, Conventional vehicles, Average speed, Travel time, Delay

\section{Introduction}

The significant development of the smart driving technology is expected to alter the perception of transportation and the Autonomous Vehicles (AVs) are becoming the next generation of transportation modes. In the past few years automation technology has gone through a huge development from semiautonomous vehicles to fully autonomous vehicles. Major vehicles' manufacturers and IT companies are investing billions of dollars to produce and develop the next generation of vehicles (Hao 2017).

Within UAE, Dubai is taking a big leap in the adaption of artificial intelligence and becoming the world largest laboratory for technology, research and

\footnotetext{
*Graduate Student, American University of Sharjah, United Arab Emirates.

${ }^{ \pm}$Professor, American University of Sharjah, United Arab Emirates.
} 
development. Dubai has launched a future transportation strategy to transform $25 \%$ of Dubai's transportation to autonomous modes by 2030 (WAM 2016).

There are many challenges that are expected to face the implementation of this technology. One of the major challenges that are expected to happen is the interaction between AVs and Conventional Vehicles (CVs) in the traffic fleet, using the same roads. This is expected to happen at the early stages of the adoption, as the market penetration rate, for AVs (percentage of AVs) would not be $100 \%$. The AVs will start with a small market penetration rates and later the market penetration rates will increase gradually until it ultimately reaches $100 \%$. The purpose of this study is to evaluate the impact of AVs on freeway traffic performance at different market penetration rates (percentage of AVs in the traffic flow) values and at two different congestion levels (0.6 and 0.8 demand to capacity ratios).

The paper consists of five sections that are organized as follows:

- Section 1 provides a brief introduction about AVs and the research conducted in this paper.

- Section 2 presents a literature and background review about AVs definition, advantages and disadvantages.

- Section 3 discusses the methodology regarding the modelling of AVs and scenarios that were considered in this research.

- Section 4 shows the results obtained from the simulation runs, discussion and analyses of the results

- Finally, section 5 summarizes the research outcomes and includes recommendations for future work

\section{Literature Review}

This section provides a review of the existing relevant researches on AVs regarding their potential impacts on different aspects.

\section{Definition and Levels of Autonomous Vehicles}

AVs are defined as self-driving or driverless vehicles that can operate without a human driver to control driving tasks such as steering, braking, deceleration and acceleration, or monitor the roadway constantly. AVs use a combination of sensors such as radar, computer vision, LIDAR, sonar, GPS, odometry and inertial measurement units to perceive their surroundings. Sensory information is interpreted by advanced control systems for path navigation and identification of obstacles and relevant signage (Verre 2018).

According to the Society of Automotive Engineering (SAE 2018), automation levels are classified into six levels; with level 0 represents no automation (CVs) and level 5 shows a full automation, which is the considered level of automation in this study (i.e., level 5). 


\section{Benefits of Autonomous Vehicles}

AVs have several advantages that include the safety, sustainability, efficiency, convenience, social benefits, traffic performance and environmental impacts. In addition, they are expected to eliminate human errors that can cause accidents such reducing greenhouse gases emissions (Simon et al. 2015). According to the National Highway Traffic Safety Administration (NHTSA), 94\% of crash accidents are due to human errors, and more than 35,000 people died in serious accidents in U.S. in 2015. So, AVs can save a lot of lives by eliminating accidents caused by human errors (NHTSA 2018).

AVs are equipped with many sensors in addition to vehicle to vehicle (V2V), vehicle to infrastructure (V2I) communications and driver assistance technologies such as Adaptive Cruise Control (ACC) and Intelligent Cruise Control (ICC). These technologies can positively impact the traffic performance as vehicles that are equipped with these technologies can track the leading vehicle and estimate the speed and distance differences. Based on that, ACC automatically accelerates or decelerates the vehicle to maintain a safe headway at desired speed and prevents rear-end collisions. This can reduce or optimize gaps between vehicles on the road and results in a smooth and safe traffic flow. It was found that a small percentage of vehicles equipped with these technologies results in a drastic reduction in traffic congestion, and thus increases traffic stability and road capacity (Kesting et al. 2007, Ioannou and Chien 1993, Aria et al. 2016, Tientrakool et al. 2011, Arnaout and Bowling 2011). This is consistent with the findings of the report Co-published by World Economic Forum and Boston consulting group in 2018. The results of the reports revealed that AVs are expected to cut urban travel time by $4 \%$

In addition, AVs reduce the parking spaces needed in the city by $43.5 \%$. As AVs can parks on their own after dropping the users at any remote parking lots outside the city. Also, they can park more closely to each other.

Furthermore, AVs can have social impacts by providing independent mode of transportation for disabled and elder people. In addition, they can reduce the stress on the drivers and increase the utility of in-vehicle time by enabling the passengers to do other activities during travel time rather than driving tasks (Litman 2014, Das et al. 2017).

The inclusion of AVs in an urban traffic fleet and its impact of AVs on urban air quality have been evaluated in a recent research. The results revealed that the increase of AVs market penetration rates in traffic fleet was associated with a decrease in Nitrogen Oxides (NOx) and Carbon Dioxide $\left(\mathrm{CO}_{2}\right)$ concentration in the air. Thus, AVs have the potential to improve urban air quality (Rafael et al. 2020).

\section{Disadvantages and Risks of AVs}

Although the implementation of AVs is expected to have many positive impacts, but there are also some negative potential impacts. The interaction between AVs and CVs and automation failure are examples of these possible negative impacts. In addition, there are some technical obstacles and risks 
associated with the AVs. Such obstacles must be overcome to ensure that the AVs are safe to be used by travelers for daily travel. Testing AVs is used to reveal any risks or errors associated with these vehicles. The increase of automation level can lead to overreliance on automation that will degrade driving skills. This could be dangerous in case of system failure (Strand et al. 2014). Moreover, AVs can encourage people to travel more and thus increase vehicle miles' travel (VMT) and greenhouse emissions since they can provide an easy, comfortable and safe mode of transportation. In addition, added population of new travelers of elderly and disabled people can also increase VMT and greenhouse emissions (Bierstedt et al. 2014).

The limitations and risks of Automated Driving System (ADS) have been evaluated in a recent research work. It was concluded that a compromise between human driving and ADS is better to avoid the risks associated with ADS and get the maximum benefits of ADS (Bocca and Baek 2020).

\section{Barriers to Implementation}

AVs' cost is one of the major barriers to large-scale market adoption of these vehicles. AVs have technology needs that include sensors, communication software and guidance technologies that increase their cost besides engineering and computing requirements. These software and hardware costs make AVs unaffordable for most of the people. It is expected that the cost of AVs will decrease with mass production and large-scale adoption similar to any other technological advances. In addition, some advocates claim that fuel, insurance and parking-cost savings will partly offset these incremental costs (Fagnant and Kockelman 2015). This can make their price affordable over time and increase their ownership. In addition, lacking nationally recognized licensing and liability standards for AVs is considered as a main barrier to the implementation of AVs. Furthermore, there is a major concern on how to develop a robust system for AVs to protect their data and minimize the exposure to risk as they may be targeted or hacked by computer hackers or terrorists and manipulated remotely. Such actions can cause many problems such as accidents or traffic disruption or terrorism acts (Fagnant and Kockelman 2015).

\section{Methodology}

The microsimulation software VISSIM was used to build a simulation model of a mixed traffic of AVs and CVs in order to evaluate the impacts of AVs on traffic performance. The considered network consists of a $10-\mathrm{km}$ stretch of a major freeway in Dubai (E 311) with five lanes in each direction and six junctions (two right-in-right-out junctions, a single point interchange and two full-cloverleaf junctions with additional ramps). The capacity of each lane is assumed to be 2000 vehicles per hour. Thus, the capacity of road is equal 10000 vehicles per hour in each direction. In addition, the posted speed is $110 \mathrm{Kph}$ and the speed limit is 130 Kph. 
AVs and CVs exhibit different driving behaviors that should be modeled to accurately simulate each type of vehicles. The driving behavior in microscopic traffic simulation is governed by several functions (such as; car following and lane changing behavior). Car following behavioral models control vehicle's longitudinal speed, acceleration and the gap between the lead vehicle and the following vehicle. Lane changing model determines when it is acceptable to change lanes and how to do so. The default driving behavior in VISSIM was used as a driving behavior model for CVs. However, some parameters in the default driving behavior were modified in order to model the presence of AVs in VISSIM. Using the VISSIM model, the impacts of AVs were evaluated at two demand to capacity ratios of 0.6 which corresponds to level of service $\mathrm{C}$, and 0.8 which corresponds to level of service $\mathrm{D}$. These two ratios are selected to represent under saturated conditions.

In addition, the impacts were evaluated at nine different market penetration rates (percentages) of AVs. The percentages of AVs were considered to be $5 \%$ to $25 \%$ with increments of 5\%, to represent the early stages adoption and from $40 \%$ to $100 \%$ with $20 \%$ increment.

In total 18 scenarios were simulated. Each scenario was simulated for 5 runs using different random seed numbers, then the trimmed average of the three middle values for each scenario were considered as the average result (Aria et al. 2016, Ngan et al. 2004).

\section{Modeling AVs}

As mentioned before AVs have different capabilities and performance than CVs. AVs can be modeled in VISSIM through internal model interface and external interfaces as shown in Figure 1.

The presence of AVs was modeled by adjusting the default driving behavior parameters used in VISSIM to satisfy the main characteristics of AVs. VISSIM enables users to customize driving behavior parameters such as (car following, lane changing, lateral behavior and reaction to signal controls).

Although AVs driving behavior is under development, and there is no standard driving behavior model of AVs, in this research, AVs driving behavior model parameters are adjusted based on literature review.

Based on the general understanding of the driving behavior of AVs, it can be described as follows:

- AVs keep smaller standstill distance.

- AVs keep smaller headway.

- AVs keep the desired speed strictly (without a distribution).

- AVs accelerate \& decelerate equally (without a distribution). 
Figure 1. AVs Modeling in PTV VISSIM (PTV Group, 2017)

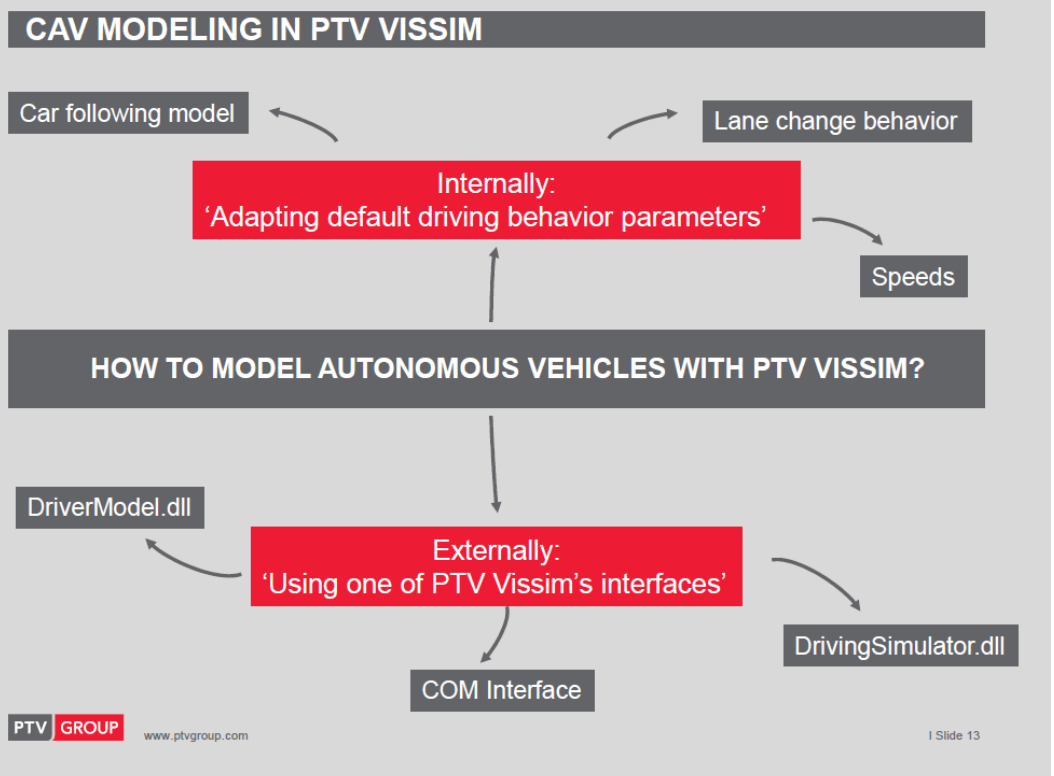

\section{Results and Discussion}

The impact of AVs on freeway traffic performance was evaluated at different market penetration rates $(0 \%, 5 \%, 10 \%, 15 \%, 20 \%, 25 \%, 40 \%, 60 \%, 80 \%$, and $100 \%)$ and at two different demand to capacity ratios (0.6 and 0.8). Some statistical analyses were conducted to interpret the results obtained from simulation runs. Descriptive statistics such as trimmed average and standard deviation for each scenario were calculated. Moreover, t-test was carried out to test the hypotheses that were assumed and to examine the significance of the difference between scenarios.

\section{Results Relative to Different Market penetration rates of AVs}

In this section, the performance of both AVs and CVs (in terms of average speed, travel time and delay) for each demand to capacity ratio is evaluated at each AVs market penetration rates scenario. The numerical values, reported in this section, are the percent improvements or reductions for each demand to capacity ratio at different market penetration rates scenarios, compared to the baseline scenario, which is $0 \%$ AVs. Because of the lack of available data, the base model is not calibrated against real-life data. Therefore, the values of speed, travel time and delay are not of much interest and the numerical values, reported in this section, are the percentage of the increase or reduction for each market penetration rates scenario, compared to the baseline scenario, which is $0 \%$ AVs (i.e. 100\% $\mathrm{CVs})$. 
It should be noted that at $100 \% \mathrm{AVs}$ the traffic fleet consists of AVs only and no CVs exist in the network. Therefore, the discussion of average speed, travel time and delay will be for the AVs only.

\section{$\underline{\text { Average Speed }}$}

The trimmed average of the speeds for AVs and CVs for 0.6 and 0.8 ratios were obtained at different market penetration rates scenarios. When the demand to capacity ratio is set to be 0.6 or 0.8 , the average speeds for AVs and CVs are obtained for all AVs market penetration rates s, as summarized in Tables 1 and 2.

Table 1. Percentage of Average Speed Improvement for AVs

\begin{tabular}{|c|c|c|}
\hline $\begin{array}{c}\text { AVs market penetration } \\
\text { rates }\end{array}$ & $\mathbf{0 . 6}$ & $\mathbf{0 . 8}$ \\
\hline $\mathbf{5 \%}$ & 4.5 & 4.3 \\
\hline $\mathbf{1 0 \%}$ & 5.0 & 4.9 \\
\hline $\mathbf{1 5 \%}$ & 5.3 & 5.5 \\
\hline $\mathbf{2 0 \%}$ & 5.5 & 6.1 \\
\hline $\mathbf{2 5 \%}$ & 5.9 & 6.8 \\
\hline $\mathbf{4 0 \%}$ & 6.8 & 8.3 \\
\hline $\mathbf{6 0 \%}$ & 7.9 & 10.4 \\
\hline $\mathbf{8 0 \%}$ & 8.8 & 12.5 \\
\hline $\mathbf{1 0 0 \%}$ & 9.7 & 14.5 \\
\hline
\end{tabular}

Table 2. Percentage of the Average Speed Improvement for CVs

\begin{tabular}{|c|c|c|}
\hline $\begin{array}{c}\text { AVs market penetration } \\
\text { rates }\end{array}$ & $\mathbf{0 . 6}$ & $\mathbf{0 . 8}$ \\
\hline $\mathbf{5 \%}$ & 0.2 & 0.5 \\
\hline $\mathbf{1 0 \%}$ & 0.6 & 1.0 \\
\hline $\mathbf{1 5 \%}$ & 0.7 & 1.3 \\
\hline $\mathbf{2 0 \%}$ & 1.1 & 1.7 \\
\hline $\mathbf{2 5 \%}$ & 1.4 & 2.3 \\
\hline $\mathbf{4 0 \%}$ & 1.9 & 3.4 \\
\hline $\mathbf{6 0 \%}$ & 2.8 & 5.1 \\
\hline $\mathbf{8 0 \%}$ & 4.0 & 6.8 \\
\hline
\end{tabular}

From Tables 1 and 2, the average speed for both AVs and CVs increased as AVs market penetration rate increased from 5\% to $100 \%$. When comparing the results of AVs and CVs it can be noted that AVs have higher improvement percentages than CVs. The improvement for $\mathrm{AVs}$ at 0.6 demand to capacity ratio ranges from about $5 \%$ with $5 \%$ AVs to about $10 \%$ with $100 \%$ AVs. On the other hand, the improvement for CVs ranges from about 0.2 with $5 \%$ AVs to around $4 \%$ with $80 \%$ AVs.

Moreover, noted form the two tables that improvement percentages increased with the increase of the demand to capacity ratio from 0.6 to 0.8 . The percentage of improvement for AVs increased from about $10 \%$ to about $15 \%$ for AVs (at 
$100 \% \mathrm{AVs}$ ) and from about $4 \%$ to about $7 \%$ for CVs (at $80 \% \mathrm{AVs}$ ), which is consistent with Aria et al.'s (2016) findings that the positive impacts of AVs will be more efficient and highlighted when the network becomes more crowded.

\section{$\underline{\text { Travel Time }}$}

Considering 0.6 and 0.8 demand to capacity ratios, the simulation results for the travel times for AVs and CVs, are discussed in this section. The comparisons in this section show the percentage of reduction in travel time, relative to the $0 \%$ AVs. For demand to capacity ratios of 0.6 and 0.8 , the results are presented in Tables 3 and 4.

Table 3. Percentage of Travel Time Reduction for AVs

\begin{tabular}{|c|c|c|}
\hline $\begin{array}{c}\text { AVs market penetration } \\
\text { rates }\end{array}$ & $\mathbf{0 . 6}$ & $\mathbf{0 . 8}$ \\
\hline $\mathbf{5 \%}$ & 0.33 & 3.7 \\
\hline $\mathbf{1 0 \%}$ & 0.67 & 4.5 \\
\hline $\mathbf{1 5 \%}$ & 1.2 & 5.4 \\
\hline $\mathbf{2 0 \%}$ & 1.3 & 5.6 \\
\hline $\mathbf{2 5 \%}$ & 1.96 & 5.8 \\
\hline $\mathbf{4 0 \%}$ & 3.5 & 7.1 \\
\hline $\mathbf{6 0 \%}$ & 5.1 & 8.6 \\
\hline $\mathbf{8 0 \%}$ & 6.6 & 10.1 \\
\hline $\mathbf{1 0 0 \%}$ & 8.1 & 11.7 \\
\hline
\end{tabular}

Table 4 Percentage of Travel Time Reduction for CVs

\begin{tabular}{|c|c|c|}
\hline $\begin{array}{c}\text { AVs market penetration } \\
\text { rates }\end{array}$ & \multicolumn{2}{|c|}{ Demand to capacity ratio } \\
\hline $\mathbf{5 \%}$ & 0.07 & $\mathbf{0 . 8}$ \\
\hline $\mathbf{1 0 \%}$ & 0.26 & 0.57 \\
\hline $\mathbf{1 5 \%}$ & 0.5 & 0.2 \\
\hline $\mathbf{2 0 \%}$ & 0.48 & 1.1 \\
\hline $\mathbf{2 5 \%}$ & 0.90 & 1.6 \\
\hline $\mathbf{4 0 \%}$ & 1.8 & 2.1 \\
\hline $\mathbf{6 0 \%}$ & 2.8 & 2.7 \\
\hline $\mathbf{8 0 \%}$ & 3.4 & 4.1 \\
\hline
\end{tabular}

Similar to what was observed in average speed results, Tables 3 and 4 show that the travel time improved as the market penetration rates of AVs increased from $5 \%$ to $100 \%$. At 0.6 demand to capacity ratio the travel time for AVs decreased by $8 \%$ with $100 \%$ AVs while the reduction in travel time for CVs is about $3.4 \%$ with $80 \%$ AVs. The reduction in travel time increased when the demand to capacity ratio increased from 0.6 to 0.8 . The maximum reduction in travel time for AVs was obtained at 0.8 demand to capacity ratio with $100 \% \mathrm{AVs}$ (about $12 \%$ ), while the maximum reduction for CVs is about $5.5 \%$ with $80 \%$ AVs.

The reduction of travel time associated with the presence of AVs in the traffic is due to the fact the AVs travel with a smaller time headway (THW), compared to 
CVs. This, in turn, increases the number of gaps in the network, and thus decreases the travel time of vehicles.

Delay

The delay is calculated by subtracting the free flow travel time (when the vehicles travel with posted speed) from observed travel time (when the vehicles travel with a speed less than posted speed). In addition, the ratios shown in the delay comparisons are the percentage of reduction in delay, relative to the $0 \%$ AVs. The delay data for AVs and CVs are depicted in Tables 5 and 6 respectively.

Table 5. Percentage of Delay Reduction for AVs

\begin{tabular}{|c|c|c|}
\hline $\begin{array}{c}\text { AVs market penetration } \\
\text { rates }\end{array}$ & \multicolumn{2}{|c|}{ Demand to capacity ratio } \\
\hline $\mathbf{5 \%}$ & 17.96 & $\mathbf{0 . 8}$ \\
\hline $\mathbf{1 0 \%}$ & 25.6 & 10.4 \\
\hline $\mathbf{1 5 \%}$ & 30.8 & 17.1 \\
\hline $\mathbf{2 0 \%}$ & 34.1 & 21.5 \\
\hline $\mathbf{2 5 \%}$ & 40.6 & 27.2 \\
\hline $\mathbf{4 0 \%}$ & 54.4 & 33.3 \\
\hline $\mathbf{6 0 \%}$ & 71.1 & 45.4 \\
\hline $\mathbf{8 0 \%}$ & 84.1 & 63.2 \\
\hline $\mathbf{1 0 0 \%}$ & 96.5 & 79.1 \\
\hline
\end{tabular}

Table 6 Percentage of Delay Reduction for CVs

\begin{tabular}{|c|c|c|}
\hline $\begin{array}{c}\text { AVs market penetration } \\
\text { rates }\end{array}$ & $\mathbf{0 . 6}$ & $\mathbf{0 . 8}$ \\
\hline $\mathbf{5 \%}$ & 3.5 & 4.9 \\
\hline $\mathbf{1 0 \%}$ & 9.3 & 9.6 \\
\hline $\mathbf{1 5 \%}$ & 12.7 & 12.8 \\
\hline $\mathbf{2 0 \%}$ & 16.95 & 16.5 \\
\hline $\mathbf{2 5 \%}$ & 22.4 & 22.4 \\
\hline $\mathbf{4 0 \%}$ & 33.2 & 32.3 \\
\hline $\mathbf{6 0 \%}$ & 49.5 & 47.7 \\
\hline $\mathbf{8 0 \%}$ & 59.7 & 61.3 \\
\hline
\end{tabular}

According to Tables 5 and 6 the delay decreased (improved) as the market penetration rates of AVs increased. The delay reduction percentages for AVs at 0.6 demand to capacity ratio ranges from about $18 \%$ with $5 \%$ AVs to about $97 \%$. The reductions for CVs range from $3.5 \%$ to about $60 \%$ at AVs percentage of $5 \%$ and $80 \%$, respectively. At 0.8 demand to capacity ratio, the reduction percentages for AVs range from $10 \%$ with $5 \%$ AVs to about $95 \%$ with $100 \%$ AVs while the reduction for $\mathrm{CVs}$ ranges from $5 \%$ with $5 \%$ AVs to $62 \%$ with $80 \%$ AVs.

This reduction in delay resulted in a reduction in travel time that was observed in the previous section. However, the percentages of improvement for delay are much higher than travel time percentages because delay values are smaller in 
magnitude, so any small improvement in delay time results in a significant percentage improvement in delay.

t-test

In this section, a test of hypotheses is conducted (using the t-test) to check that the variations of traffic performance measures are not random variations in the data, and that they are due to the difference between distributions. Two types of ttest were conducted; dependent and independent t-tests. Two hypotheses tests are formed and evaluated.

The Null hypothesis $\left(H_{0}\right)$ assumes that there is no difference between the means of the two populations or scenarios (Devore et al. 2013).

$$
H_{0}: \mu_{1}=\mu_{2}
$$

While the Alternative hypothesis $\left(H_{a}\right)$ assumes that there a difference exist between the two means (Devore et al. 2013).

$$
H_{a}: \mu_{1} \neq \mu_{2}
$$

Where:

$\mu_{1}$ s the mean of the first scenario.

$\mu_{2}$ s the mean of the second scenario.

$\Delta=$ the difference between means.

When conducting the t-test, two possible errors may occur. Type I error, which is rejecting $H_{0}$ when it is actually true and Type II error which is accepting $H_{0}$ when it is false.

The probability of making type I error is denoted by " $\alpha$ " and is called level of significance. In this test procedure $\alpha=0.05$ will be used.

\section{$\underline{\text { Dependent t-test }}$}

Dependent (paired) t-tests were carried out to compare the average speed of each market penetration rates scenario to determine if there is a statistical difference in average speed between consecutive scenarios or not. First the following equation was used to calculate $t$ value.

$$
\mathrm{t}=\frac{\bar{d}-\Delta}{\frac{s_{d}}{\sqrt{n}}},
$$

where,

$\mathrm{n}=$ number of pairs

$\bar{d}=$ the mean difference

$\Delta=$ the difference between means

$s_{d}=$ standard deviation of the difference 
Then P-value was determined from $\mathrm{t}$ curves with $\mathrm{n}-1$ degree of freedom. Based on the P-value obtained the null hypothesis is accepted or rejected as follows:

if P-value $\leq \alpha$ reject $H_{0}$ if P-value $>\alpha$ accept $H_{0}$

- At 0.6 demand to capacity ratio

Table 7 shows the p-values and the decision of accepting or rejecting the null hypothesis.

Table 7. t-test Results for Comparing Scenarios' Speeds at 0.6 Demand to Capacity Ratio

\begin{tabular}{|c|c|c|}
\hline Scenarios & P-value & Decision \\
\hline $\mathbf{0 \%}$ and 5\% & $2.56 \mathrm{E}-04$ & reject $H_{0}$ \\
\hline $\mathbf{5 \%}$ and 10\% & $1.42 \mathrm{E}-04$ & reject $H_{0}$ \\
\hline $\mathbf{1 0 \%}$ and 15\% & $2.55 \mathrm{E}-03$ & reject $H_{0}$ \\
\hline $\mathbf{1 5 \%}$ and 20\% & $2.50 \mathrm{E}-04$ & reject $H_{0}$ \\
\hline $\mathbf{2 0 \%}$ and $\mathbf{2 5 \%}$ & $4.50 \mathrm{E}-04$ & reject $H_{0}$ \\
\hline $\mathbf{2 5 \%}$ and 40\% & $09.52 \mathrm{E}-06$ & reject $H_{0}$ \\
\hline $\mathbf{4 0 \%}$ and 60\% & $4.50 \mathrm{E}-05$ & reject $H_{0}$ \\
\hline $\mathbf{6 0 \%}$ and $\mathbf{8 0 \%}$ & $6.67 \mathrm{E}-08$ & reject $H_{0}$ \\
\hline $\mathbf{8 0 \%}$ and $\mathbf{1 0 0 \%}$ & $4.57 \mathrm{E}-07$ & reject $H_{0}$ \\
\hline
\end{tabular}

According to Table 7, all p-values are significantly less than $\alpha(0.05)$. Therefore, the null hypothesis is rejected and there is a statistical difference between the average speeds of each scenario. This means that the average speed improved significantly with the increase of AVs market penetration rates from $0 \%$ to $100 \%$.

- At 0.8 demand to capacity ratio

Table 8 contains the P-values at 0.8 demand to capacity ratio.

Table 8. t-test Results for Comparing Scenarios' Speeds at 0.8 Demand to Capacity Ratio

\begin{tabular}{|c|c|c|}
\hline Scenarios & P-value & Decision \\
\hline $\mathbf{0 \%}$ and 5\% & $3.71 \mathrm{E}-03$ & reject $H_{0}$ \\
\hline $\mathbf{1 0 \%}$ and 10\% & $2.32 \mathrm{E}-03$ & reject $H_{0}$ \\
\hline $\mathbf{1 5 \%}$ and 20\% & $1.75 \mathrm{E}-04$ & reject $H_{0}$ \\
\hline $\mathbf{2 0 \%}$ and 25\% & $3.45 \mathrm{E}-03$ & reject $H_{0}$ \\
\hline $\mathbf{2 5 \%}$ and 40\% & $5.73 \mathrm{E}-04$ & reject $H_{0}$ \\
\hline $\mathbf{4 0 \%}$ and 60\% & $1.32 \mathrm{E}-04$ & reject $H_{0}$ \\
\hline $\mathbf{6 0 \%}$ and $\mathbf{8 0 \%}$ & $1.66 \mathrm{E}-05$ & reject $H_{0}$ \\
\hline $\mathbf{8 0 \%}$ and 100\% & $8.59 \mathrm{E}-08$ & reject $H_{0}$ \\
\hline & $1.62 \mathrm{E}-05$ & reject $H_{0}$ \\
\hline
\end{tabular}


Table 8 shows that there is a significant difference between each market penetration rates average speeds at 0.8 demand to capacity ratio as all the $\mathrm{P}$-values are much smaller than $\alpha$ so the null hypothesis is rejected similar to 0.6 case any change in AVs market penetration rates yields a significant difference in average speed.

\section{$\underline{\text { Independent t-test }}$}

The independent (Pooled Variance) t-test was conducted to compare the means of the speed values of AVs and CVs in the same scenario (the same market penetration rate) to determine whether there is a significant difference between $\mathrm{AVs}$ and $\mathrm{CVs}$ speed values using the following equation:

$$
\mathrm{t}=\frac{\bar{x}_{1}-\bar{x}_{2}}{\sqrt{\frac{s_{1}^{2}}{n_{1}}+\frac{s_{2}{ }^{2}}{n_{2}}}}
$$

where,

$\bar{x}_{1}$ : mean of group1

$\bar{x}_{2}:$ mean of group2

$s_{1}$ : sample variance of group1

$s_{2}$ : sample variance of group 2

$n_{1}:$ number of observations of group 1

$n_{2}:$ number of observations of group 2

- At 0.6 demand to capacity ratio

Independent t-test was conducted between AVs and CVs average speeds, and P-values were calculated and summarized in Table 9.

Table 9. $t$-test between $C V$ s and AVs Speeds at 0.6 Demand to Capacity Ratio

\begin{tabular}{|c|c|c|}
\hline Scenarios & P-value & Decision \\
\hline $\mathbf{5 \%}$ & $1.9266 \mathrm{E}-08$ & reject $H_{0}$ \\
\hline $\mathbf{1 0 \%}$ & $3.59779 \mathrm{E}-09$ & reject $H_{0}$ \\
\hline $\mathbf{1 5 \%}$ & $1.98112 \mathrm{E}-08$ & reject $H_{0}$ \\
\hline $\mathbf{2 0 \%}$ & $2.05817 \mathrm{E}-08$ & reject $H_{0}$ \\
\hline $\mathbf{2 5 \%}$ & $5.01216 \mathrm{E}-09$ & reject $H_{0}$ \\
\hline $\mathbf{4 0 \%}$ & $1.40731 \mathrm{E}-09$ & reject $H_{0}$ \\
\hline $\mathbf{6 0 \%}$ & $3.17224 \mathrm{E}-12$ & reject $H_{0}$ \\
\hline $\mathbf{8 0 \%}$ & $7.26973 \mathrm{E}-09$ & reject $H_{0}$ \\
\hline
\end{tabular}

According to Table 9, there is a significant difference between AVs and CVs speed values at all market penetration rates as the congestion at this demand to capacity ratio is small, so AVs can travel freely with speeds significantly higher than CVs.

- At 0.8 demand to capacity ratio 

10.

The P-values at 0.8 demand to capacity ratio are obtained and shown in Table

Table 10. $t$-test between $C V s$ and AVs Speeds at 0.8 Demand to Capacity Ratio

\begin{tabular}{|c|c|c|}
\hline Scenarios & P-value & Decision \\
\hline $\mathbf{5 \%}$ & $1.23986 \mathrm{E}-07$ & reject $H_{0}$ \\
\hline $\mathbf{1 0 \%}$ & $8.15699 \mathrm{E}-08$ & reject $H_{0}$ \\
\hline $\mathbf{1 5 \%}$ & $1.41604 \mathrm{E}-07$ & reject $H_{0}$ \\
\hline $\mathbf{2 0 \%}$ & $5.60178 \mathrm{E}-08$ & reject $H_{0}$ \\
\hline $\mathbf{2 5 \%}$ & $6.14618 \mathrm{E}-07$ & reject $H_{0}$ \\
\hline $\mathbf{4 0 \%}$ & $2.82743 \mathrm{E}-08$ & reject $H_{0}$ \\
\hline $\mathbf{6 0 \%}$ & $7.85416 \mathrm{E}-12$ & reject $H_{0}$ \\
\hline $\mathbf{8 0 \%}$ & $4.085 \mathrm{E}-09$ & reject $H_{0}$ \\
\hline
\end{tabular}

Similar to 0.6 case, there is a significant difference between the means of AVs and CVs speed values for the same reason that AVs travel much faster than CVs at low congestion.

\section{Conclusions}

Autonomous vehicles are the next generation vehicles that have drawn significant attention recently and it has become a major concern to major cities. As the use of AVs is an essential component for smart city. Dubai is planning to adopt this new technology as part of its transportation system's transformation process. However, the introduction of this technology is expected to have various impacts. These impacts should be studied in order to evaluate and maximize the benefits of these vehicles and minimize the risks and errors associated with them. The purpose of this research is to determine how the adoption of AVs can impact the traffic performance of Dubai's freeways.

The microsimulation software VISSIM was utilized to develop microsimulation model of a $10-\mathrm{km}$ section of a major freeway in Dubai. The purpose of the simulation is to evaluate different scenarios that represent different market penetration rates $\mathrm{s}$ of AVs and different congestion levels. This study considered various market penetration rates s for AVs $(0 \%, 5 \%, 10 \%, 15 \%, 20 \%$, $25 \%, 40 \%, 60 \%, 80 \%$, and $100 \%$ ). It should be noted that the $0 \%$ was used as a benchmark for comparison purposes. Different traffic congestion levers were evaluated by considering two demand to capacity ratios of 0.6 and 0.8 , representing undersaturated conditions. In summary, the results showed that:

1) Increasing AVs fleet percentage yields an increase in average speed and a decrease in travel time and delay, as CVs are replaced with AVs. This can be attributed to the fact that AVs strictly keep a constant speed without variation and travel with a smaller THW. Therefore, this provides smooth and uniform traffic flow in the network. 
2) The performance improvement increases as the demand to capacity ratio increases, which is consistent with (Aria et al. 2016) findings that positive effects of AVs are highlighted when the congestion increases and when the traffic flow becomes denser.

3) The highest improvements were achieved at 0.8 demand to capacity ratio with $100 \%$ AVs. The average speed increased by about $15 \%$, the travel time decreased by about $12 \%$. However, the maximum reduction in delay was achieved at 0.6 demand to capacity ratio (around 97\%) which is slightly higher than the delay reduction at 0.8 demand to capacity ratio (about 94\%).

4) The comparison between scenarios, using dependent t-test, revealed that any change in $\mathrm{AVs}$ market penetration rates yields a significant difference in the traffic performance, at 0.6 and 0.8 demand to capacity ratios.

5) By comparing the mean of the average speeds of AVs and CVs, using independent t-test, it was noted that there is a significant difference between AVs and CVs speed values at all market penetration rates for both 0.6 and 0.8 demand to capacity ratios.

This research considered only the impacts of AVs on Freeway performance without any considerations of changing the lane width or changing any of the road characteristics. Therefore, future work can consider the following:

- Implementing the procedure followed in this paper for over saturated traffic conditions to achieve results that cover all possible real-life situations, especially during peak hours.

- Safety on both Freeways and intersections

- AVs' system failure and how they will perform in this case

- Performance of AVs on arterial streets' networks dealing with signalized junctions and roundabouts

\section{References}

Aria E, Olstam J, Schwietering C (2016) Investigation of automated vehicle effects on driver's behavior and traffic performance. Transportation Research Procedia 15: 761-770.

Arnaout G, Bowling S (2011) Towards reducing traffic congestion using cooperative adaptive cruise control on a freeway with a ramp. Journal of Industrial Engineering and Management 4(4): 699-717.

Bierstedt J, Gooze A, Gray C, Peterman J, Raykin L, Walters J (2014) Effects of nextgeneration vehicles on travel demand and highway capacity. FP Think Working Group 8.

Bocca A, Baek D (2020) Automated driving systems: key advantages, limitations and risks. IEEE Conference Publication. Retrieved from: https://bit.ly/394dnYp. [Accessed 20 February 2020].

Das S, Sekar A, Chen R, Kim H, Wallington T, Williams E (2017) Impacts of autonomous vehicles on consumers time-use patterns. Challenges 8(2). 
Devore J, Farnum N, Doi J (2013) Applied statistics for engineers and scientists. $3^{\text {rd }}$ Edition. Belmont, Calif: Duxbury.

Fagnant J, Kockelman K (2015) Preparing a nation for autonomous vehicles: opportunities, barriers and policy recommendations. Transportation Research Part A 77(Jul): 167-181.

Hao K (2017) At least 47 cities around the world are piloting self-driving cars. Retrieved from: https://bit.ly/2T0O5EW. [Accessed 20 February 2020].

Ioannou A, Chien C (1993) Autonomous intelligent cruise control. IEEE Transactions on Vehicular Technology 42(4): 657-672.

Kesting A, Treiber M, Schönhof M, Kranke F, Helbing D (2007) Jam-avoiding adaptive cruise control (acc) and its impact on traffic dynamics. Traffic and Granular Flow 5(Feb): 633-643.

Litman T (2014) Autonomous vehicle implementation predictions: implications for transport planning. Transportation Research Board Annual Meeting, 36-42.

Ngan V, Sayed T, Abdelfatah A (2004) Impacts of various parameters on transit signal priority effectiveness. Journal of Public Transportation 7(3): 71-93.

NHTSA (2018) Automated vehicles for safety. Retrieved from: https://www.nhtsa.gov/ technology-innovation/automated-vehicles-safety. [Accessed 20 February 2020].

PTV Group (2017) PTV VISSIM \& connected autonomous vehicles. VISSIM User Manual.

Rafael S, Correia L, Lopes D, Bandeira J, Coelho M, Andeade M, et al. (2020) Autonomous vehicles opportunities for cities air quality. Science of the Total Environment 712(Apr).

SAE (2018) Taxonomy and definitions for terms related to driving automation systems for on-road motor vehicles (J3016 ground vehicle standard) - SAE mobilus. Saemobilus.sae.org, 2020. Retrieved from: https://saemobilus.sae.org/content/ j3016_201806. [Accessed 20 February 2020].

Simon K, Alson J, Snapp L, and Hula A (2015) Can Transportation Emission Reductions Be Achieved Autonomously? Environ. Sci. Technol., vol. 49, no. 24, pp. 1391013911.

Strand N, Nilsson J, Karlsson I, Nilsson L, (2014) Semi-automated versus highly automated driving in critical situations caused by automation failures. Transportation Research Part F: Traffic Psychology and Behaviour 27(PB): 218-228.

Tientrakool P, Ho C, Maxemchuk F (2011) Highway capacity benefits from using vehicleto-vehicle communication and sensors for collision avoidance. IEEE Vehicular Technology Conference, 0-4.

WAM (2016) Mohammed bin Rashid approves Dubai autonomous transportation strategy final. Retrieved from: http://wam.ae/en/details/1395294678474. [Accessed 20 February 2020].

World Economic Forum (2018) Reshaping urban mobility with autonomous vehicles lessons from the city of Boston. Retrieved from: http://www3.weforum.org/docs/WE F_Reshaping_Urban_Mobility_with_Autonomous_Vehicles_2018.pdf. [Accessed: 29 February 2020]. 
